Leading Article

\title{
Acute mesenteric ischaemia
}

\author{
A.P. Corder and I. Taylor
}

University Department of Surgery, Level F, Southampton General Hospital, Tremona Road, Southampton SO9 $4 X Y, U K$

Acute mesenteric ischaemia, when extensive, has an extremely high mortality. ${ }^{1,2}$ Arterial occlusion, most commonly embolic, is the commonest cause but infarction may also occur due to low flow states, mesenteric venous thrombosis and vasculitis. ${ }^{3,4}$ Extensive mesenteric ischaemia is often perceived to be an uncommon emergency ${ }^{3}$ and is not given priority in the differential diagnosis of acute abdominal pain. In reality, a hospital serving 200,000 patients may deal with one such case each month on average. ${ }^{5}$ Delay in diagnosis is of critical importance because of the rapidity with which the small bowel suffers ischaemic damage. In rats and dogs, irreversible infarction occurs 8-10 hours after complete arterial occlusion. ${ }^{6}$ Revascularization is often technically possible but has to be undertaken within this period if the small bowel is to be saved.

Clearly much rests on speedy diagnosis. Abdominal pain, usually sudden, is almost invariable in arterial occlusion. ${ }^{1,2}$ though the onset in venous occlusion or low flow states may be more insidious. Vomiting is frequent and diarrhoea, sometimes with blood, may also occur. On examination, the abdomen may be less tender than suggested by the degree of pain. Abdominal signs often become more marked after infarction has occurred but attempts at revascularization are then unlikely to be useful. Sufficiently early diagnosis is thus heavily dependent on clinical awareness and suspicion. ${ }^{3}$ This may be heightened by a knowledge of predisposing factors. Arterial occlusions are associated with cardiac arrhythmias, valvular disease, previous emboli, myocardial infarction, congestive cardiac failure and hypotension. ${ }^{3,7}$ Other factors include hypovolaemia, diuretic use, vasoconstrictors and digitalis therapy. ${ }^{3,8}$ Venous occlusions are associated with portal hypertension, states of hypercoagulation and intra-abdominal malignancy. ${ }^{9}$

Correspondence: A.P. Corder.

Received: 17 July 1992
Blood tests are of limited diagnostic use. The white cell count is often grossly raised and the amylase often moderately so. Despite early optimism, ${ }^{10}$ hyperphosphataemia has not been confirmed to be a useful marker of mesenteric infarction." Radiological changes are minimal until infarction has occurred but it is worth noting that gas in the portal vein, once thought to be uniformly lethal has been followed by recovery. ${ }^{12}$

Once a provisional diagnosis has been made, the question of the use of angiography arises. The advantages include the possible definitive diagnosis of embolism, thrombosis, low flow states or mesenteric vein thrombosis, the effective exclusion of mesenteric ischaemia as a diagnosis and the possibility of vasodilator, lytic or angioplasty therapy. ${ }^{9,7,13}$ The disadvantages include a high rate of negative examinations, ${ }^{14}$ possible critical delay in the surgical removal of emboli and the possibility of misdiagnosis in the presence of an abnormal mesenteric circulation which is not producing ischaemia. ${ }^{15}$ Angiography is unlikely to be helpful once infarction has occurred. Its true place in the management of acute mesenteric ischaemia will not be evident until it has been used more frequently in the very early stages of mesenteric ischaemia.

At present, the mainstay of treatment is urgent operation. Embolism is probably the commonest cause of massive small bowel ischaemia. ${ }^{5,16}$ Patients who have known risk factors for embolism (particularly prosthetic heart valves and atrial fibrillation) and who develop sudden abdominal pain should be regarded as having small bowel embolism until proven otherwise. Angiography in this situation may delay operation but may also save an unnecessary laparotomy or indicate the need for a suitably experienced surgeon to be present in theatre. If laparotomy can be performed before irreversible ischaemia occurs, embolectomy may give excellent results. ${ }^{16-18}$ The best results are obtained using a proximal arteriotomy ${ }^{19}$ but this approach requires care and skill. For less experienced surgeons who are unable to get help, embolectomy may be carried out via the ileo-colic artery ${ }^{20}$ but this does not deal with the problem of propagated thrombus. 
The problem of thrombosis associated with occlusive disease at the origin of the superior mesenteric artery is much more difficult and carries a higher mortality. In some cases, occlusive disease also affects the coeliac axis and the inferior mesenteric artery. When the superior mesenteric artery finally becomes occluded, infarction of parts of the fore and hind gut may also occur. Such catastrophic events may be preceded by a period of 'mesenteric angina'. ${ }^{20}$ Revascularization during this chronic phase may give excellent long-term results. ${ }^{21}$ Once acute ischaemia develops, the mortality is extremely high. ${ }^{5,16,17}$ If extensive gangrene has not developed, acute revascularization from the aorto-iliac to the superior mesenteric system may be attempted either directly or using autologous saphenous vein. ${ }^{3,4}$ Successful revascularization in the acute phase using angioplasty has also been reported. ${ }^{13}$

Laparotomy offers no benefits, in the early stages, to those patients with mesenteric ischaemia due to low flow states associated with critical illness. Such states have been successfully treated by selective angiography and vasodilator therapy delivered into the superior mesenteric artery. Obviously however, there are practical difficulties in performing adequate angiography on patients who are being managed in the intensive care unit.

Mesenteric venous thrombosis is rarely recognized before laparotomy by which time infarction has usually occurred. There are isolated reports of successful thrombectomy ${ }^{22}$ but this approach is unlikely to be generally useful.

Sadly, the great majority of patients presenting with acute mesenteric ischaemia will do so with overt gangrene of the intestine. When embolism is the cause, the proximal jejunal branches may be spared leaving a short segment of viable proximal jejunum with a pulsatile arterial supply. Although infarction may extend to the mid transverse colon, resection is very worthwhile and has in many cases been followed by a good quality of life without the need for long-term parenteral nutrition. ${ }^{23}$ Other cases, probably due to occlusive disease, in which infarction involves the whole midgut (and occasionally includes the foregut and hindgut) are essentially untreatable. Cases of less extensive infarction also occur in which the benefits of resection are more immediately obvious.

There is sometimes doubt about the viability of segments of intestine, whether or not they have been reperfused. The mucosa is far more sensitive to ischaemia than the serosa and therefore even minor serosal changes are usually accompanied by irreversible mucosal damage. Intra-operative Doppler assessment and assessment by intravenous fluorescein with a Wood's lamp have both been advocated as methods for assessing the viability of dubious intestine. The latter seems to be the more useful. ${ }^{24}$ When the problem relates to the viability of a resection margin, it is easily solved by opening the bowel along the anti-mesenteric border and viewing the mucosa directly.

Following extensive resections of ischaemic bowel, some authorities regard primary anastomosis as unsafe. ${ }^{4}$ Often, however, especially in cases of extensive embolic infarction, the cut-off between viable and non-viable bowel is quite clear and the bowel ends are obviously well perfused. Primary anastomosis in these cases makes sense. If the proximal small bowel is exteriorized in these cases, an extremely high output fistula is formed. This delays the introduction of enteral nutrition which in turn delays small intestinal adaptation. Large fluid losses condemn the patient to prolonged intravenous therapy whether or not parenteral nutrition is required full time. Clearly, however, exteriorization may be sensible after any resection for low output states in which adequate anastomotic perfusion cannot be guaranteed. If there is doubt about the viability of the remaining gut, a 'second look' procedure should be carried out after about 24 hours.

Whatever the future may hold for advances in diagnosis and therapy, the high mortality of extensive mesenteric ischaemia will only be decreased by increased clinical awareness. Treatment of mesenteric angina may forestall some cases of catastrophic mesenteric infarction. Earlier recognition and treatment of the majority who present without previous symptoms is required to prevent massive intestinal infarction. These points are stressed in the paper by Heys and colleagues in this issue of the Journal. ${ }^{25}$

\section{References}

1. Krausz, M.M. \& Manny, J. Acute superior mesenteric artery occlusion: a plea for early diagnosis. Surgery 1978, 83: 482-485.

2. Andersson, R., Parsson, H., Isaksson, B. \& Norgren, L. Acute intestinal ischaemia. A 14 year retrospective investigation. Acta Chir Scand 1984, 150: 217-221.

3. Williams, L.F. Mesenteric ischemia. Surg Clin North Am 1988, 68: $331-353$.

4. Marston, A. Small bowel ischaemia. Current Pract Surg 1990, 2: $146-154$.

5. Wilson, C., Gupta, R. \& Gilmour, D.G. Acute superior mesenteric ischaemia. Br J Surg 1987, 74: 279-281.

6. Gharagozloo, F., Bulkley, G.B., Zuidema, G.D., O’Mara, C.S. \& Alderson, P.O. The use of intraperitoneal xenon for early diagnosis of acute mesenteric ischaemia. Surgery 1984, 95: 404-411.

7. Boley, S.J., Sprayragen, S., Siegelman, S.S. \& Vieth, F.J. Initial results from an aggressive approach to acute mesenteric ischaemia. Surgery 1977, 82: 848-855. 
8. Gottlieb, J.E., Menashe, P.I. \& Cruz, E. Gastrointestinal complications in critically ill patients: the intensivists' overview. Am J Gastroenterol 1986, 81: 227-238.

9. Witte, C.L., Brewer, M.L., Witte, M.H. et al. Protean manifestations of pyelothrombosis. Ann Surg 1985, 202: 191-202.

10. Jamieson, W.G., Marchuk, S., Rowsom, J. \& Durand, D. The early diagnosis of massive acute intestinal ischaemia. $\mathrm{Br} J$ Surg 1982, 69(Suppl): S52-S53.

11. Mosley, J.G. \& Marston, A. Splanchnic Ischaemia and Multiple Organ Failure. Edward Arnold, London, Baltimore, 1989, p. 282.

12. Kranendonk, S.E., Bruining, H.A. \& van Urk, H. Survival after portal venous gas due to mesenteric vascular occlusion. Br J Surg 1983, 70: 183-184.

13. VanDiense, W.H., Zawacki, J.K. \& Phillips, D. Treatment of acute mesenteric ischaemia by percutaneous transluminal angioplasty. Gastroenterology 1986, 91: 745-748.

14. Clark, R.A. \& Gallant, T.E. Acute mesenteric ischaemia: angiographic spectrum. Am J Roentgen 1984, 142: 555-562.

15. Croft, R.J., Menon, G.P. \& Marston, A. Does intestinal angina exist? A critical study of obstructed visceral arteries. Br J Surg 1981, 68: 316-318.

16. Bergen, J.J., Dean, R.H., Conn, J. \& Yao, J.S.T. Revascularization in treatment of mesenteric infarction. Ann Surg 1975, 182: 430-438.

17. Ottinger, L.W. The surgical management of acute occlusion of the superior mesenteric artery. Ann Surg 1978, 188: $721-733$.
18. Clavien, P.A. Diagnosis and management of mesenteric infarction. Br J Surg 1990, 77: 601-603.

19. Ogborn, N. \& Pories, W.J. Reconstruction of the mesenteric and coeliac arteries. In: Rob, C. \& Smith, R. Operative Surgery. Vascular Surgery, 4th ed. Butterworths, London, 1985 , pp. $158-159$.

20. Kwaan, H.M. \& Connolly, J.E. Prevention of intestinal infarction resulting from mesenteric arterial occlusive disease. Surg Gynecol Obstet 1983, 157: 321-324.

21. Marston, A., Clarke, J.M.F., Garcia Garcia, J. \& Miller, A.L. Intestinal function and intestinal blood supply: a 20 year surgical study. Gut 1985, 26: 656-666.

22. Ghaly, M. \& Frawley, J.E. Superior mesenteric vein thrombosis. Aust NZ J Surg 1986, 56: 277-279.

23. Sitges-Serra, A., Mas, X., Roqueta, F., Figueras, J. \& Sanz, F. Mesenteric infarction: an analysis of 83 patients with prognostic studies in $\mathbf{4 4}$ cases undergoing a massive small bowel resection. Br J Surg 1988, 75: 544-548.

24. Bulkley, G.B., Zuidema, G.D., Hamilton, S.R., O'Mara, C.S., Klacsmann, P.G. \& Horn, S.D. Intraoperative determination of small intestinal viability following ischaemic injury. Ann Surg 1981, 193: 628-637.

25. Heys, S.D., Brittenden, J. \& Crofts, T.J. Acute mesenteric ischaemia: the continuing difficulty in early diagnosis. Postgrad Med J 1993, 69: 48-51. 